\title{
SFCNS -
}

\section{une fédération à multiples facettes}

\section{Karl Schaller}

Pr Dr méd., Genève, Président de la SFCNS

SFCNS a été créé en 2009 dans le but de promouvoir la collaboration interdisciplinaire et interprofessionnelle des différentes disciplines des neurosciences cliniques en Suisse. Lors de sa création, la fédération comptait six sociétés de médecine représentant leurs disciplines respectives et visant à promouvoir les neurosciences cliniques dans leur ensemble. A l'issue du premier congrès SFCNS qui s'est déroulé à Bâle en 2010, plusieurs autres sociétés de neurosciences ont rejoint la fédération. Aujourd'hui, pour son 10ème anniversaire, la fédération comprend 14 organisations de plus de 2000 membres individuels. Nous pouvons donc affirmer sans hésitation, qu'aujourd'hui, en 2019, une grande majorité des sociétés médicales et des spécialistes actifs dans le domaine du diagnostic et du traitement des maladies du système nerveux en Suisse font partie de notre fédération.

\section{Au service des patients victimes d'un AVC}

Depuis sa création il y a dix ans, notre fédération a déjà initié plusieurs projets contribuant à renforcer la coopération entre les neurosciences cliniques en faveur d'une meilleure qualité des soins. Dès 2011, l'organe décisionnel de la Convention intercantonale relative à la médecine hautement spécialisée (CIMHS) a décidé de confier à la SFCNS la certification, partout en Suisse, des centres de compétence pour le traitement des AVC ischémiques

\section{4th SFCNS Congress et SFCNS Imaging Course}

Le quatrième congrès de la SFCNS aura lieu à Lausanne du 23 au 25 octobre 2019. Les 14 sociétés membres de la SFCNS sont impliquées dans l'organisation de ce congrès qui n'a lieu que tous les trois ans.

Le $4^{\mathrm{e}}$ congrès de la SFCNS, présidé par le professeur Karl-Olof Lövblad de Genève, est le plus grand congrès de Suisse dans le domaine des neurosciences cliniques et constitue une plateforme d'échange unique et ce pas seulement pour les spécialistes de neurodisciplines. Pendant les trois jours du congrès, des thèmes purement médicaux tels que les douleurs dorsales ou les maux de tête, la neuro-immunologie, la neuro-inflammation seront abordés et examinés du point de vue des différentes disciplines représentées. D'autre part, des questions sur des sujets techniques dits "sensibles" tels que l'intelligence artificielle, la robotique, la réalité virtuelle et diverses technologies mobiles ainsi que leur lien avec les neurosciences seront également au cœur des manifestations du congrès. A l'issue du congrès SFCNS, le premier des six modules du SFCNS Imaging Course aura également lieu à Lausanne. Développé par le professeur Christoph Stippich de Zurich, le cours interdisciplinaire SFCNS, Imaging Course, a pour vocation d'approfondir les connaissances des participants en matière d'imagerie par résonance magnétique dans différents contextes. Plus de détails sur le congrès sur sfcns2019.congress-imk.ch et les thrombolyses. A ce jour, deux commissions interdisciplinaires et interprofessionnelles de la SFCNS, spécialement mandatées, ont d'ores et déjà certifié des unités spécialisées dans le traitement des AVC (Stroke Units) dans 13 hôpitaux. Ces deux mêmes commissions ont également certifié dans dix centres hospitaliers des Stroke Centers qui reprennent le concept des Stroke Units en l'élargissant à des services spécialisés structurels, de neuroradiologie et de neurochirurgie.

\section{Young Clinical Neuroscientists Network (YouCliN)} Lors de la troisième édition du congrès SFCNS en 2016, la jeune génération de neurocliniciens a créé sa propre organisation au sein de la fédération: le Young Clinical Neuroscientists Network (YouCliN). Il décerne cette année pour la première fois le prix YouCliN aux meilleurs travaux de recherche indépendants dans le domaine des neurosciences cliniques. Par ailleurs, YouCliN organise avec succès, de manière autonome ou en collaboration avec d'autres organisations de jeunes membres des manifestations de formation continue ou postgrade.

\section{Notre propre revue en ligne}

Tous les départements spécialisés de la SFCNS ainsi que YouCliN publient régulièrement des articles dans le Clinical and Translational Neuroscience. Plus de 50 exemples de cas, travaux originaux, éditoriaux et reviews de nombreux auteurs ont, à ce jour, été soumis, évalués par des pairs et publiés sur le site internet de la revue. En tant que revue officielle de plusieurs sociétés suisses de neuroscience, réalisée sous l'égide de la SFCNS et accessible au public gratuitement sur internet, cette revue s'adresse à tous les acteurs des neurosciences cliniques en Suisse et à l'étranger.

Pour notre fédération à caractère interdisciplinaire, la collaboration internationale revêt une importance grandissante. Ainsi, aujourd'hui, la SFCNS entretient de plus en plus de coopérations internationales avec des chercheurs en sciences fondamentales, coordonne les questions de recherche sur le plan transnational et contribue ainsi à la promotion des neurosciences cliniques au niveau paneuropéen.

Vous trouverez de plus amples informations sur la fédération sur le site sfcns.ch 